\title{
LINC00365-SCGB2A1 axis inhibits the viability of breast cancer through targeting NF-kB signaling
}

\author{
LICHAO ZHANG $^{1 *}$, XIAOYU YAN ${ }^{1 *}$, SIHANG YU $^{1}$, XINRU ZHONG ${ }^{1}$, \\ RUI TIAN $^{1}$, LONG XU ${ }^{1}$, XUEHAI BIAN ${ }^{2}$ and JING SU ${ }^{1}$ \\ ${ }^{1}$ Department of Pathophysiology, College of Basic Medical Sciences, Jilin University; \\ ${ }^{2}$ Department of Thyroid Surgery, China-Japan Union Hospital of Jilin University, Changchun, Jilin 130012, P.R. China
}

Received March 19, 2019; Accepted November 1, 2019

DOI: $10.3892 / 01.2019 .11166$

\begin{abstract}
Breast cancer is the most common high-grade malignancy in women. The lack of therapeutic targets has limited the treatment of breast cancer. Recently, long noncoding RNAs (lncRNAs) have been demonstrated to be dysregulated in various types of cancer. However, the specific mechanisms by which lncRNAs influence breast cancer have remained largely unclear. To bridge this research gap, the present study focused on the lncRNA LINC00365, which is expressed at a low level in breast cancer. Secretoglobin family 2A member 1 (SCGB2A1) was identified as a potential target protein regulated by LINC00365. The results of the present study demonstrated that the overexpression of LINC00365 and SCGB2A1 inhibited cell viability and induced cell apoptosis through the inhibition of the NF- $\kappa \mathrm{B}$ signaling pathway in breast cancer cells. These findings indicated that LINC00365 may serve a crucial role in breast cancer and may be considered as a novel target for the clinical treatment of breast cancer.
\end{abstract}

\section{Introduction}

Breast cancer is one of the leading causes of death among women (1). The high heterogeneity of breast tumor cells prevents traditional treatment options from satisfying the individualized treatment needs of patients with breast cancer. Genetic alteration is a feature of breast cancer; if molecular-targeted therapy was

Correspondence to: Professor Jing Su, Department of Pathophysiology, College of Basic Medical Sciences, Jilin University, 126 Xinmin Street, Changchun, Jilin 130012, P.R. China

E-mail: sujing@jlu.edu.cn

Professor Xuehai Bian, Department of Thyroid Surgery, China-Japan Union Hospital of Jilin University, 126 Xiantai Street, Changchun, Jilin 130012, P.R. China

E-mail: bianxh@jiu.edu.cn

${ }^{*}$ Contributed equally

Key words: long non-coding RNA, secretoglobin family 2A member $1, \mathrm{NF}-\mathrm{\kappa B}$, apoptosis, viability, breast cancer developed based on different genetic abnormalities in patients, the mortality rates could be decreased (2). With the development of gene sequencing and bioinformatics, the association between the regulation of non-coding RNA sequences in the cancer genome and the complex biological characteristics of tumors has become a hot topic of research. Our previous study demonstrated that the long noncoding RNA (lncRNA) LINC00365 and the secreted protein secretoglobin family $2 \mathrm{~A}$ member 1 (SCGB2A1; also termed mammaglobin B are expressed at low levels in gastric cancer, as revealed by bioinformatics analysis, and that there is a regulatory relationship between them (3). Expression of SCGB2A1 is associated with chemotherapy and radiation resistance and cancer cell stemness (4). Therefore, the present study aims to determine the association between LINC00365 and SCGB2A1 in breast cancer and to develop new targets for breast cancer treatment.

SCGB2A1 is a small secreted protein that is a member of the superfamily of uterine proteins (5). The gene encoding this protein is located on chromosome 11q12.2 (6). SCGB2A1 was first isolated by Becker et al in 1998 (7); it has been identified in epithelial cells in the lung, breast, salivary gland, sweat gland and prostate and is closely related to cell secretion, inflammation, tissue repair and tumorigenesis (8). SCGB2A1 is considered a candidate marker for detecting certain minimal cancers in lymph nodes and for diagnosing tumor cells hidden in the exudate of patients with various malignancies (4). In addition, gene expression profiling identified SCGB2A1 as a highly expressed gene in all histological types of ovarian cancer (9). Previous studies have reported that SCGB2A1 is expressed at a low level in luminal breast cancer compared with that in normal tissue $(10,11)$, but the specific mechanism behind its involvement in this disease remains unclear. Long non-coding RNAs (lncRNAs) are a group of non-protein-coding RNAs that are $>200$ nucleotides long $(12,13)$. Due to their complex spatial structure, the mechanisms involved in regulating their expression are particularly diverse and complex. Characterization of the functional mechanisms of lncRNA effects in tumors not only contributes to the application of clinical biomarkers, but also promotes the development of new cancer therapeutic targets (14). A number of lncRNAs have been demonstrated to regulate important cancer-related processes (15), including apoptosis, viability, metastasis, metabolism and chemotherapy resistance $(16,17)$. LINC00365 is one of the lncRNAs encoded 
by a gene with a chromosomal location $13 q 12.3$ (18). Our previous study revealed that LINC00365 exhibits significantly different expression levels in gastric cancer compared with those in normal tissue (3). In addition, studies using bioinformatics approaches have predicted that SCGB2A1 secreted into the blood and urine is a potential target for LINC00365 (3).

The activation of nuclear transcription factor $\kappa B$ $(\mathrm{NF}-\kappa \mathrm{B})$ is involved in the transcriptional regulation of many genes (19). The role of the $N F-\kappa B-m e d i a t e d$ cell signal transduction pathway in cell viability and apoptosis has been a focus of intensive research globally (20-22). $\mathrm{NF}-\kappa \mathrm{B}$ suppresses apoptosis by inducing the expression of apoptosis-inhibitory genes, including inhibitors of apoptosis proteins (IAPs), cellular FLICE-like inhibitory protein, TNF receptor-associated factor 1 (TRAF1) and TRAF2 (22-25). Two typical pro-survival NF- $\kappa \mathrm{B}$ targets are X-linked inhibitor of apoptosis and $\mathrm{Bcl} 2$-like 1 (Bcl-xl), which can block apoptosis at multiple steps $(26,27)$. Similarly, NF- $\kappa$ B can promote tumor cell viability by regulating TNF- $\alpha$, chemokines, adhesion factors, transforming growth factors and other molecules involved in various stages of the inflammatory response (28). Previous findings have demonstrated that $\mathrm{NF}-\kappa \mathrm{B}$ is overexpressed in multiple types of breast cancer cells (29), but the specific mechanism associated with this process remains to be identified (30). Based on a literature review and previous studies, it is hypothesized in the present study that LINC00365 and SCGB2A1 may affect the activity of breast cancer cells by affecting the transcriptional activity of NF- $\mathrm{NB}$.

The present study aimed to investigate the underlying mechanism of LINC00365 and SCGB2A1 in breast cancer. In addition, the LINC00365-SCGB2A1 axis was demonstrated to participate in the viability and apoptosis of breast cancer cells by regulating the $\mathrm{NF}-\kappa \mathrm{B}$ signaling pathway. The results of the present study suggested that LINC00365 and SCGB2A1 may become promising targets for breast cancer treatment.

\section{Materials and methods}

Tissue collection. Paired breast cancer and paracancerous (3-5 cm distal from the cancer tissue) tissues were collected from 30 female patients (age range, 35-70 years) who underwent surgical resection at the China-Japan Union Hospital of Jilin University (Table I). Approval for this study was provided by the Ethics Committee of the China-Japan Union Hospital of Jilin University. The patients were not treated locally or systemically prior to surgery. All tissues were washed with sterile phosphate-buffered saline, snap-frozen in liquid nitrogen and stored at $-80^{\circ} \mathrm{C}$ until RNA extraction.

Cell lines and culture. Human invasive ductal carcinoma cell lines MCF-7 and T47D were cultured in RPMI-1640 medium (Gibco; Thermo Fisher Scientific, Inc.) supplemented with $10 \%$ fetal bovine serum (FBS) (Gibco; Thermo Fisher Scientific,Inc.), $100 \mathrm{IU} / \mathrm{ml}$ penicillin and $100 \mathrm{mg} / \mathrm{ml}$ streptomycin (Invitrogen; Thermo Fisher Scientific, Inc.). Normal human breast epithelial MCF-10A cells were cultured in F12 Ham's Mixture (Gibco; Thermo Fisher Scientific, Inc.) supplemented with 5\% FBS, epidermal growth factor, insulin, hydrocortisone, cholera toxin,
$100 \mathrm{IU} / \mathrm{ml}$ penicillin and $100 \mathrm{mg} / \mathrm{ml}$ streptomycin (Invitrogen; Thermo Fisher Scientific, Inc.). All cells were incubated at $37^{\circ} \mathrm{C}$ in a humidified atmosphere with $5 \% \mathrm{CO}_{2}$.

Expression of LINC00365 and SCGB2A1. Short hairpin RNA (shRNA) sequences were constructed by Shanghai Genechem Co., Ltd. Full-length human LINC00365 and SCGB2A1 expression vectors were constructed by subcloning full-length LINC00365 or SCGB2A1 cDNA fragments into pcDNA3.1 vectors (Shanghai Genechem Co., Ltd.). Transfections were performed using Lipofectamine ${ }^{\circledR} 2000$ (Invitrogen; Thermo Fisher Scientific, Inc.) according to the manufacturer's protocol. Briefly, MCF-7 and T47D cells were seeded into 6 -well plates at the density of $6 \times 10^{5}$ cells per well and transfected with $4 \mu \mathrm{g}$ LINC00365 or SCGB2A1 expression vector for $6 \mathrm{~h}$ with $10 \mu \mathrm{l}(1 \mu \mathrm{g} / \mu \mathrm{l})$ Lipofectamine ${ }^{\circledR} 2000$ per well. After $48 \mathrm{~h}$, the cells were harvested for subsequent experiments.

Cell viability assay. Cell viability was determined by real-time unlabeled cell assay (RTCA). MCF-7 and T47D cells transfected with an empty vector, LINC00365 or SCGB2A1 overexpression vectors were inoculated into an E-Plate assay plate at a density of $1.2 \times 10^{4}$ cells/well and subsequently maintained at room temperature for $30 \mathrm{~min}$. The E-Plate detection was placed on a test stand, and a cell viability curve was created following real-time dynamic detection of cell viability.

$R N A$ extraction and reverse transcription-quantitative $P C R$ $(R T-q P C R)$ analysis. Total RNA was extracted from tissues or cells using TRIzol ${ }^{\circledR}$ reagent (Invitrogen; Thermo Fisher Scientific, Inc.) and reverse-transcribed into cDNA according to the manufacturer's instructions, by using the EasyScript First-Strand cDNA Synthesis (Beijing Transgen Biotech Co., Ltd.). qPCR was performed using an MX300P instrument (Agilent Technologies, Inc.), followed by a three-step PCR protocol. cDNA was used as template and cycling parameters were as follows: $95^{\circ} \mathrm{C}$ for $2 \mathrm{~min}$, followed by 40 cycles of $95^{\circ} \mathrm{C}$ for $10 \mathrm{sec}, 60^{\circ} \mathrm{C}$ for $30 \mathrm{sec}$ and $72^{\circ} \mathrm{C}$ for $30 \mathrm{sec}$. Relative expression was calculated by $2^{-\Delta \Delta \mathrm{Cq}}$ among different experimental groups with normalization to GAPDH expression (31). The primer sequences were as follows: LINC00365 forward, 5-TGCTATTCTATGGCTGGGCTTC-3' and reverse, 5'-ACT GTTGAATTTCCTGGATGTGTC-3'; SCGB2A1 forward, 5'-AAACTCCTGGAGGACATGGTT-3' and reverse, 5'-ACT GCTTGAATTTCCCCATAGC-3'; C-X-C motif chemokine ligand 8 (CXCL8) forward, 5'-ATGACTTCCAAGCTGGCC GTGGCT-3' and reverse, 5'-TCTCAGCCCTCTTCAAAA ACTTCTC-3'; transforming growth factor $\beta 1$ (TGF $\beta 1$ ) forward, 5'-GGATAACACACTGCAAGTGG-3' and reverse, 5'-GAGCTGAAGCAATAGTTGGTG-3'; c-IAP1 forward, 5'-GTTCAGTGGTTCTTACTCCAGC-3', reverse, 5'-ACT GTAGGGGTTAGTCCTCGAT-3'; and GAPDH forward. 5'-AGAGGCAGGGATGATGTTCTG-3' and reverse, 5'-GAC TCATGACCACAGTCCATGC-3'.

Cloneformation assay. Following transfection with LINC00365and SCGB2A1-overexpressing vectors for $12 \mathrm{~h}, \mathrm{MCF}-7$ and T47D cells were collected, counted and seeded in a 6-well plate 
Table I. Patient information.

\begin{tabular}{|c|c|c|c|c|c|c|}
\hline No. & Sex & Age, years & ER & PR & HER-2 & Type \\
\hline 1 & Female & 52 & + & + & - & Luminal B \\
\hline 2 & Female & 47 & + & + & + & Luminal B \\
\hline 3 & Female & 68 & - & - & + & $\mathrm{HER}^{+}{ }^{+}$ \\
\hline 4 & Female & 39 & + & + & + & Luminal B \\
\hline 5 & Female & 62 & + & + & + & Luminal B \\
\hline 6 & Female & 64 & + & + & + & Luminal B \\
\hline 7 & Female & 58 & + & + & - & Luminal B \\
\hline 8 & Female & 48 & - & - & - & Basal-like \\
\hline 9 & Female & 65 & + & $2+$ & + & Luminal A \\
\hline 10 & Female & 64 & - & - & + & $\mathrm{HER}^{+}{ }^{+}$ \\
\hline 11 & Female & 46 & + & + & + & Luminal B \\
\hline 12 & Female & 40 & + & + & - & Luminal B \\
\hline 13 & Female & 54 & - & - & + & $\mathrm{HER}^{+}{ }^{+}$ \\
\hline 14 & Female & 50 & + & + & - & Luminal B \\
\hline 15 & Female & 35 & + & + & + & Luminal B \\
\hline 16 & Female & 47 & + & $2+$ & + & Luminal B \\
\hline 17 & Female & 45 & + & + & + & Luminal B \\
\hline 18 & Female & 69 & + & + & + & Luminal B \\
\hline 19 & Female & 69 & + & $2+$ & + & Luminal B \\
\hline 20 & Female & 65 & + & + & + & Luminal B \\
\hline 21 & Female & 63 & + & + & + & Luminal B \\
\hline 22 & Female & 43 & + & + & - & Luminal B \\
\hline 23 & Female & 70 & + & + & - & Luminal B \\
\hline 24 & Female & 60 & + & + & + & Luminal B \\
\hline 25 & Female & 52 & - & - & + & $\mathrm{HER}^{+}{ }^{+}$ \\
\hline 26 & Female & 38 & - & - & + & $\mathrm{HER}^{+}{ }^{+}$ \\
\hline 27 & Female & 62 & + & + & - & Luminal B \\
\hline 28 & Female & 59 & + & $2+$ & - & Luminal A \\
\hline 29 & Female & 56 & + & + & + & Luminal B \\
\hline 30 & Female & 43 & + & + & + & Luminal B \\
\hline
\end{tabular}

ER, estrogen receptor; PR, progesterone receptor; HER2, human epidermal growth factor receptor 2.

at 500 cells/well. Negative control (NC) and LINC00365- and SCGB2A1-overexpressing groups were established, and experiments were performed in triplicate for each group. Following 1-week incubation with fresh medium, the cells were fixed with $4 \%$ paraformaldehyde for $15 \mathrm{~min}$ at $37^{\circ} \mathrm{C}$, in a humidified atmosphere with $5 \% \mathrm{CO}_{2}$, washed once with PBS and stained with $0.1 \%$ crystal violet for $30 \mathrm{~min}$ at room temperature. The number of cell clones was counted using a light microscope (OLYMPUS IX-71; Olympus Corporation; magnification, x10).

Flow cytometric analysis. MCF-7 and T47D breast cancer cells were seeded in 6-well plates and transfected with empty, LINC00365- or SCGB2A1-overexpressing vectors for $48 \mathrm{~h}$. Attaching cells and cells in the supernatant were collected. Each group of cells was stained with Annexin-V FITC/propidium iodide (Annexin V Apoptosis Detection Kit II; BD Biosciences). Samples were analyzed using a BD Accuri C6 flow cytometer (Becton, Dickinson and Company).
Western blot assay. MCF-7 and T47D cells were lysed with a protein extraction reagent buffer (RIPA; Beyotime Institute of Biotechnology) containing a protease inhibitor cocktail and phenylmethylsufonyl fluoride. Protein concentration was measured using the Coomassie G250 assay (Beyotime Institute of Biotechnology). Proteins $(70 \mu \mathrm{g})$ were separated by $10 \%$ SDS-PAGE and transferred to a PVDF membrane. The membrane was blocked with $10 \%$ milk and washed with TBS $+0.1 \%$ Tween-20. Subsequently, the membrane was incubated with a primary antibody (ProteinTech Group, Inc.; BCL2, cat. no. 12789-1-AP, Bcl-XL, cat. no. 26967-1-AP, Caspase 3, cat. no. 19677-1-AP, BAX, cat. no. 50599-2-Ig10268-1-AP, $\beta$-actin, cat. no. 23660-1-AP) diluted 1:1,000 overnight at $4^{\circ} \mathrm{C}$, followed by incubation with the secondary antibody (ProteinTech Group, Inc.; anti-mouse, cat. no. SA00001-1; anti-rabbit, cat. no. SA00001-2) diluted 1:1,000 at room temperature for 1-2 h. Immunodetection was performed using ECL reagent (Thermo Fisher Scientific, Inc.) and visualized by Syngene Bio Imaging (Synoptics Ltd.). ImageJ (x64) software (National Institutes of Health) was used to quantify the results.

5-Ethynyl-2'-deoxyuridine (EdU) cell viability assay. MCF-7 and T47D cells transfected with empty, LINC00365- or SCGB2A-overexpressing vectors were inoculated into 96-well plates and fixed with $4 \%$ paraformaldehyde for $20 \mathrm{~min}$ at room temperature. Diluted EdU (cat. no. C10310-1; Suzhou Ribo Life Science Co. Ltd; $50 \mu \mathrm{l}$ ) was added per well according to the manufacturer's instructions. The cells were washed with PBS, followed by DNA staining with Hoechst-33342 at room temperature for $5 \mathrm{~min}$, and the staining was observed using OLYMPUS IX-71 fluorescence microscope (magnification, $\mathrm{x} 20$ ).

Immunofluorescence and confocal microscopy. MCF-7 and T47D cells were seeded on glass slides, transfected for $48 \mathrm{~h}$, fixed with $4 \%$ paraformaldehyde for $25 \mathrm{~min}$ at room temperature, washed with $0.01 \mathrm{M}$ PBS, treated with $0.1 \%$ Triton-PBS for $7 \mathrm{~min}$ and blocked with $5 \% \mathrm{FBS}$ for $30 \mathrm{~min}$ at room temperate. P50 primary antibody (ProteinTech Group, Inc.; $\mathrm{NF}-\kappa \mathrm{B}$, cat. no. 14220-1-AP; 1:200) was added and incubated overnight at $4^{\circ} \mathrm{C}$, followed by washing with PBS and incubation with a fluorescent secondary antibody (ProteinTech Group, Inc. FITC, cat. no. SA00003-1; 1:200) for $30 \mathrm{~min}$ in the dark at room temperature. Sections were fixed with glycerol at room temperature for $30 \mathrm{~min}$ and imaged using an Olympus FV1000 confocal laser microscope (magnification, x20).

Luciferase reporter assay. MCF-7 and T47D cells were transfected with $2 \mu \mathrm{g} / \mathrm{ml} \mathrm{NF- \kappa B}-\mathrm{Luc}$ reporter plasmid and LINC00365/SCGB2A1 plasmid (Sangon Biotech Co., Ltd.) used Lipofectamine ${ }^{\circledR} 3000$ (Invitrogen; Thermo Fisher Scientific, Inc.), LINC00365 or SCGB2A1 overexpression vectors and the internal reference plasmid for $48 \mathrm{~h}$, Renilla luciferase assay substrate and firefly luciferase detection reagent were added. Renilla luciferase was used as an internal reference, and luciferase activity was measured using a dual luciferase assay kit (Promega Corporation).

Statistical analysis. Data are expressed as the mean \pm SD. SPSS 17.0 software (SPSS, Inc.) was used for statistical analysis. 

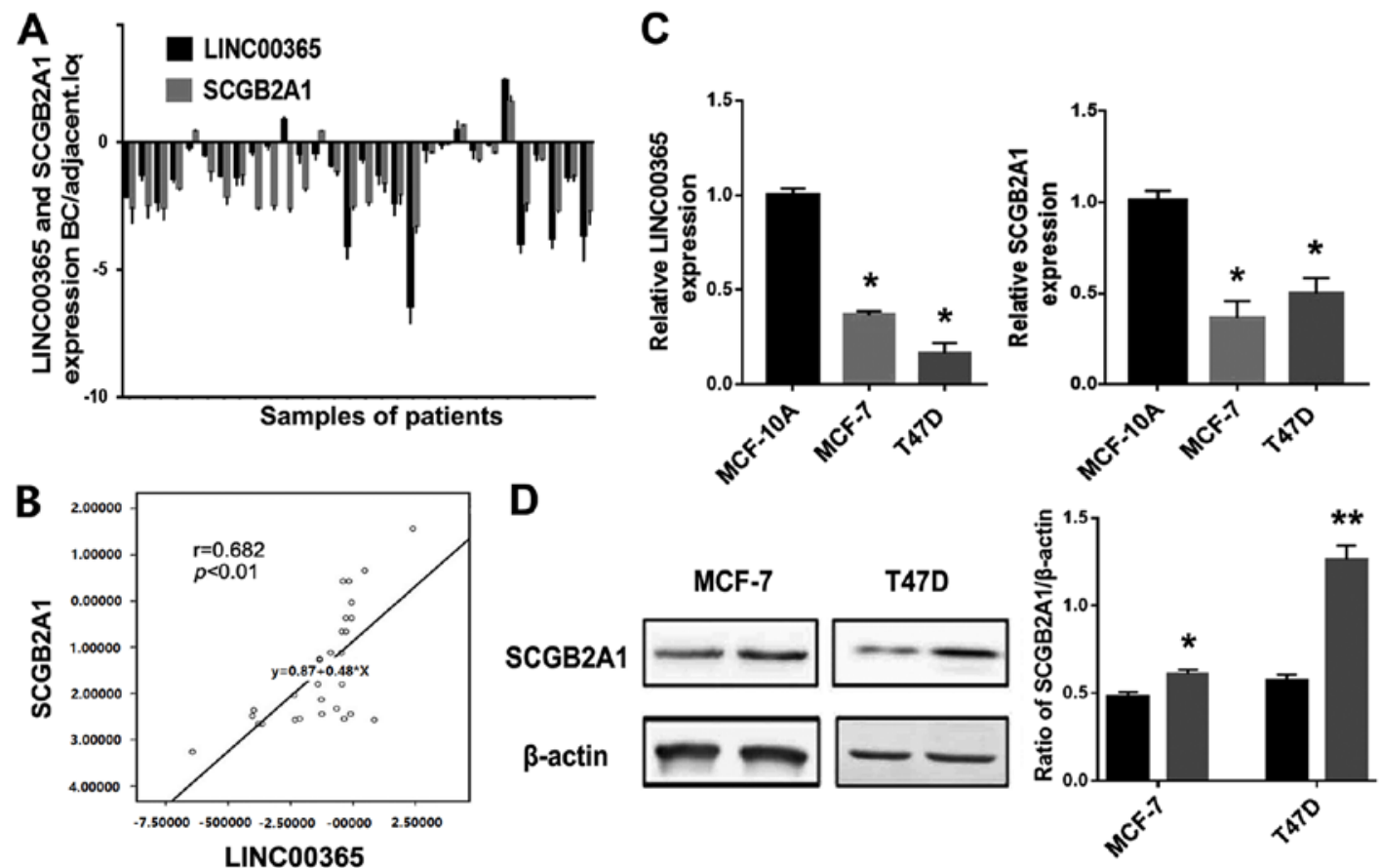

E

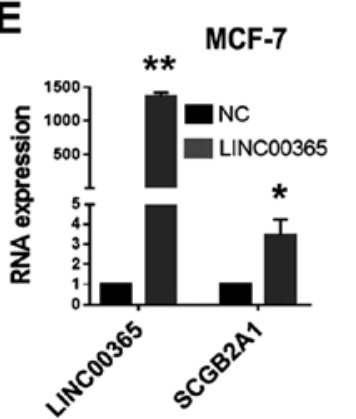

D

SCGB2A1

$\beta$-actin

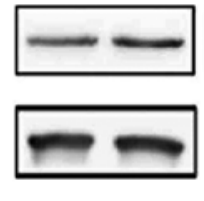

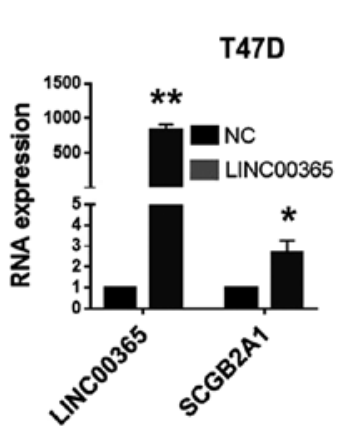

$\mathbf{F}$
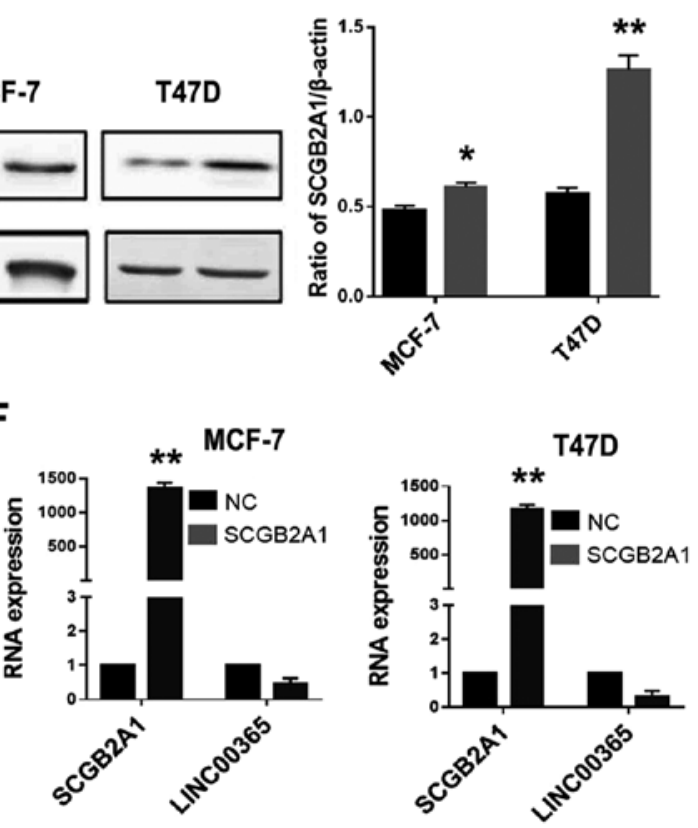

Figure 1. Expression of the long non-coding RNA LINC00365 in breast cancer tissues and cell lines. (A) LINC00365 and SCGB2A1 expression was significantly reduced in breast cancer tissues compared with the corresponding normal breast tissues in 30 patients with breast cancer. (B) Pearson's correlation analysis identified a correlation between LINC00365 and SCGB2A1 expression in breast cancer tissues. (C) Expression of LINC00365 and SCGB2A1 in breast cancer cell lines MCF-7 and T47D and a normal breast epithelial cell line MCF-10A. The expression of LINC00365 and SCGB2A1 is normalized to that of MCF-10A. (D) Increased expression of SCGB2A1 following LINC00365 overexpression in MCF-7 and T47D cell lines after $48 \mathrm{~h}$, determined by western blotting. (E) In cells overexpressing LINC00365, the expression levels of SCGB2A1 were significantly increased. (F) In cells overexpressing SCGB2A1, the expression levels of LINC00365 were not significantly altered. "P $<0.05$ and ${ }^{* *} \mathrm{P}<0.01$ vs. NC. SCGB2A1, secretoglobin family $2 \mathrm{~A}$ member 1 ; NC, negative control; qPCR, quantitative PCR.

Pearson correlation analysis was used to detect the correlation between LINC00365 and SCGB2A1 transcription level. The differences among multiple groups were analyzed using one-way ANOVA followed by Dunnett's multiple comparison post hoc test to determine statistical significance. $\mathrm{P}<0.05$ was considered to indicate a statistically significant difference.

\section{Results}

LINC00365 and SCGB2A1 exhibit low expression and are positively correlated in clinical specimens of breast cancer, as well as breast cancer cell lines. The expression levels of LINC00365 and SCGB2A1 mRNA in 30 cancer and adjacent tissues from patients with different types of breast cancer (Table I) were determined by qPCR. The results demonstrated that the expression levels of LNC00365 and SCGB2A1 were decreased in breast cancer tissues compared with adjacent tissues in the majority of patients (Fig. 1A). Pearson's correlation analysis confirmed that LINC00365 expression was positively correlated with SCGB2A1 (Fig. 1B). In addition, qPCR was used to determine the mRNA expression levels of LINC00365 and SCGB2A1 in MCF-7 and T47D human breast cancer cells, as well as MCF-10A normal breast cells. Consistently with the ex vivo results, the expression levels of LINC00365 and SCGB2A1 were lower in the two breast cancer cell lines compared with those in MCF-10A cells (Fig. 1C).

To confirm the regulatory relationship between LINC00365 and SCGB2A1, MCF-7 and T47D human breast cancer cells were transfected with a LINC00365 overexpression vector. The results demonstrated that SCGB2A1 expression was upregulated at the mRNA and protein level compared with that in the negative control group (NC) (Fig. 1D and E). However, when SCGB2A1 was overexpressed in breast cancer cells, no significant differences were observed in the change in LINC00365 expression compared with NC, which indicated 

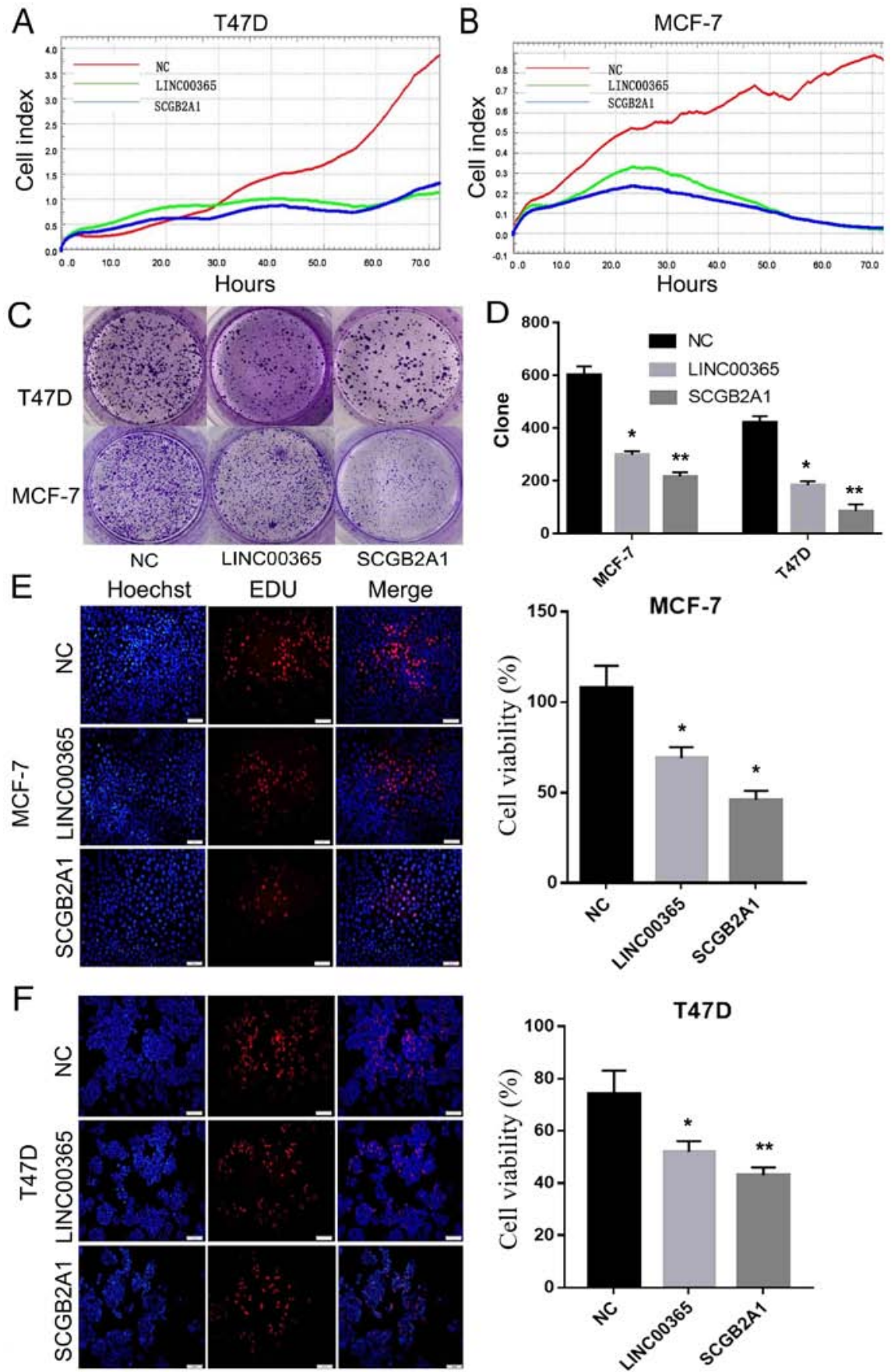

Figure 2. Overexpression of LINC00365 and SCGB2A1 inhibits the viability of breast cancer cells. (A and B) Viability of (A) T47D and (B) MCF-7 cells following LINC00365 and SCGB2A1 overexpression was detected by real-time unlabeled cell assay. (C and D) Clone formation of (C) T47D and (D) MCF-7 breast cancer cells. (E and F) EdU staining experiments demonstrated that the viability of breast cancer cells was reduced following overexpression of LINC00365 and SCGB2A1. "P<0.05 and ${ }^{* *} \mathrm{P}<0.01$ vs. NC. SCGB2A1, secretoglobin family $2 \mathrm{~A}$ member 1; NC, negative control; EdU, 5-ethynyl-2'-deoxyuridine.

that SCGB2A1 was downstream of LINC00365 in breast cancer cells (Fig. 1F).

Overexpression of LINC00365 and SCGB2Al inhibits the viability of breast cancer cells. To examine the role of LINC00365, RTCA was used to determine viability of MCF-7 and T47D breast cancer cells transfected with LINC00365 for $72 \mathrm{~h}$. The results demonstrated that overexpression of LINC00365 inhibited breast cancer cell viability by $>50 \%$ compared with that in $\mathrm{NC}$ after $72 \mathrm{~h}$. In addition, cells transfected with SCGB2A1 exhibited similar results (Fig. 2A and B).
Clone formation assay confirmed that cell viability significantly decreased following overexpression of LINC00365 or SCGB2A1 compared with that in NC (Fig. 2C and D). EdU cell viability assay results also demonstrated that overexpression of LINC00365 and SCGB2A1 significantly reduced the viability of MCF-7 and T47D breast cancer cells compared with that in the NC group (Fig. 2E and F).

Overexpression of LINC00365 and SCGB2A1 induces apoptosis in breast cancer cells. To assess the role of LINC00365 in cell death, flow cytometry was used to evaluate apoptosis in 
A
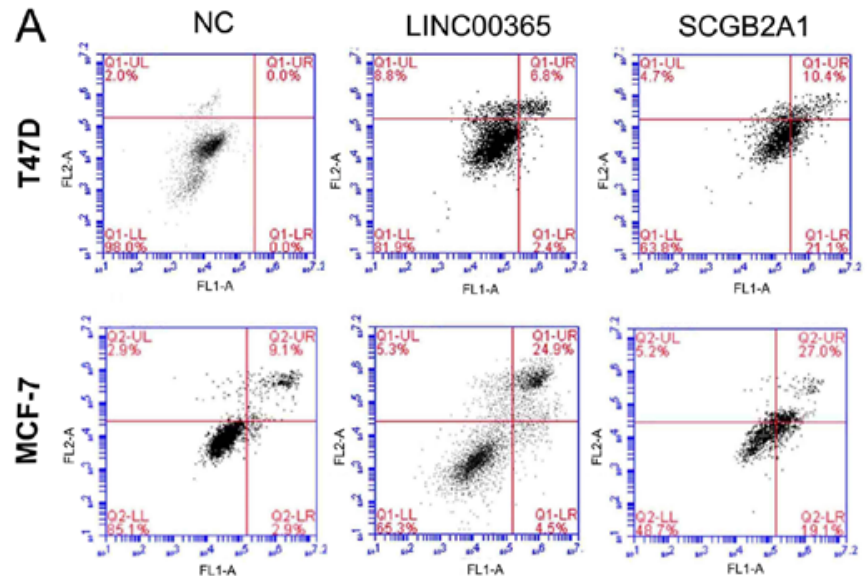

C

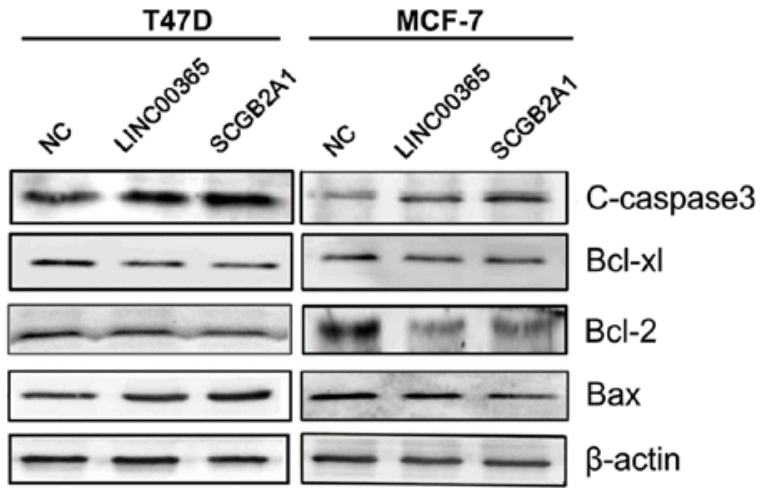

E

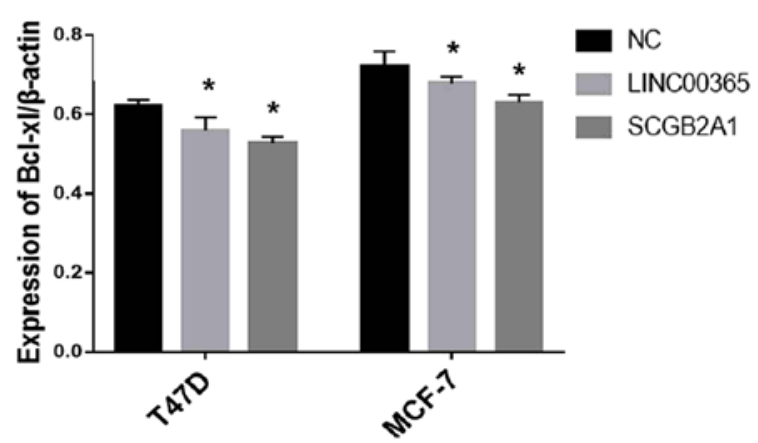

B

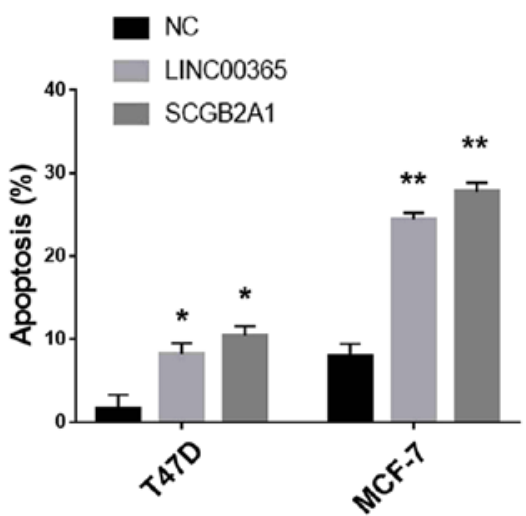

$\mathrm{D}$

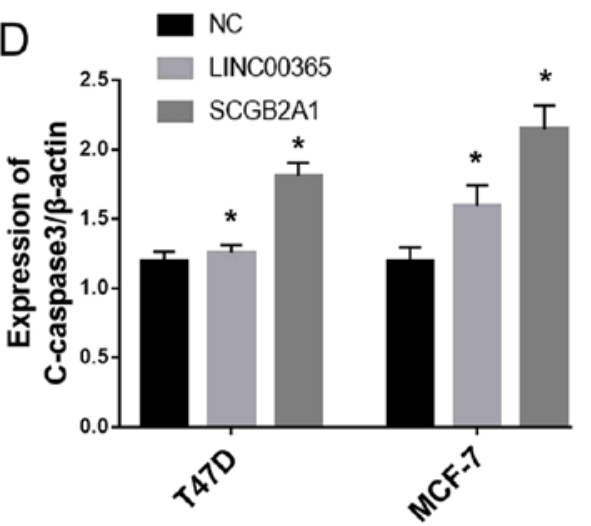

$\mathrm{F}$

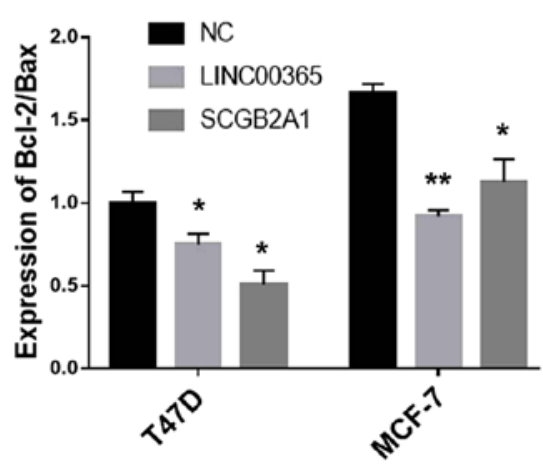

Figure 3. Overexpression of LINC00365 and SCGB2A1 induces apoptosis in breast cancer cells. (A) Apoptotic rate was determined by Annexin V/propidium iodide assay following transfection of MCF-7 and T47D cells with LINC00365- and SCGB2A1-expressing vectors. (B) Quantitative analysis of the apoptosis results. (C) Western blotting analysis of cleaved-caspase-3, Bcl-xl, Bcl-2 and Bax in MCF-7 and T47D cells following transfection with LINC00365- and SCGB2A1-expressing vectors. $\beta$-actin was used as an internal control. (D-F) The ratio of protein/ $\beta$-actin in T47D and MCF-7 cells. " $\mathrm{P}<0.05$ and ${ }^{* *} \mathrm{P}<0.01$ vs. NC. SCGB2A1, secretoglobin family 2A member 1; NC, negative control; Bcl-xl, Bcl2-like 1.

cells transfected with LINC00365 or SCGB2A1 overexpression vectors. The results demonstrated that overexpression of LINC00365 or SCGB2A1 promoted apoptosis in breast cancer cell lines MCF-7 and T47D (Fig. 3A and B). Western blotting was used to detect the expression of cleaved-caspase 3 and Bcl-2 family members, including the anti-apoptotic members Bcl-2 and Bcl-xl, as well as the pro-apoptotic member Bax. The results revealed that overexpression of LINC00365 and SCGB2A1 promoted the activation of cleaved-caspase-3 in MCF-7 and T47D cells compared with that in the NC group. In addition, the anti-apoptotic protein Bcl-xl was downregulated in MCF-7 and T47D cells, whereas Bcl-2/Bax ratio was decreased in MCF-7 and T47D cells compared with that in the NC group, indicating that overexpression of LINC00365 or SCGB2A1 induced apoptosis in MCF-7 and T47D breast cancer cells (Fig. 3C-F).

LINC00365 inhibits cell viability by suppressing the $N F-\kappa B$ signaling pathway. The levels of $\mathrm{p}-\mathrm{I} \kappa \mathrm{B} \alpha$ in breast cancer cells were determined by western blotting; the results demonstrated that LINC00365 or SCGB2A1 overexpression inhibited I $\kappa \mathrm{B} \alpha$ phosphorylation, suggesting the potential involvement of NF- $\mathrm{KB}$ signaling in breast cancer (Fig. $4 \mathrm{C}$ ). The p-I $\mathrm{KB} \alpha / \mathrm{I} \kappa \mathrm{B} \alpha$ ratio revealed that there was at least a 2 -fold difference between the MCF-7 and T47D cell lines, which indicated that NF- $\mathrm{KB}$ signaling in MCF-7 cells may be more sensitive to LINC00365 overexpression compared with that in T47D cells (Fig. 4D). In addition, immunofluorescence was used to detect the expression 
A

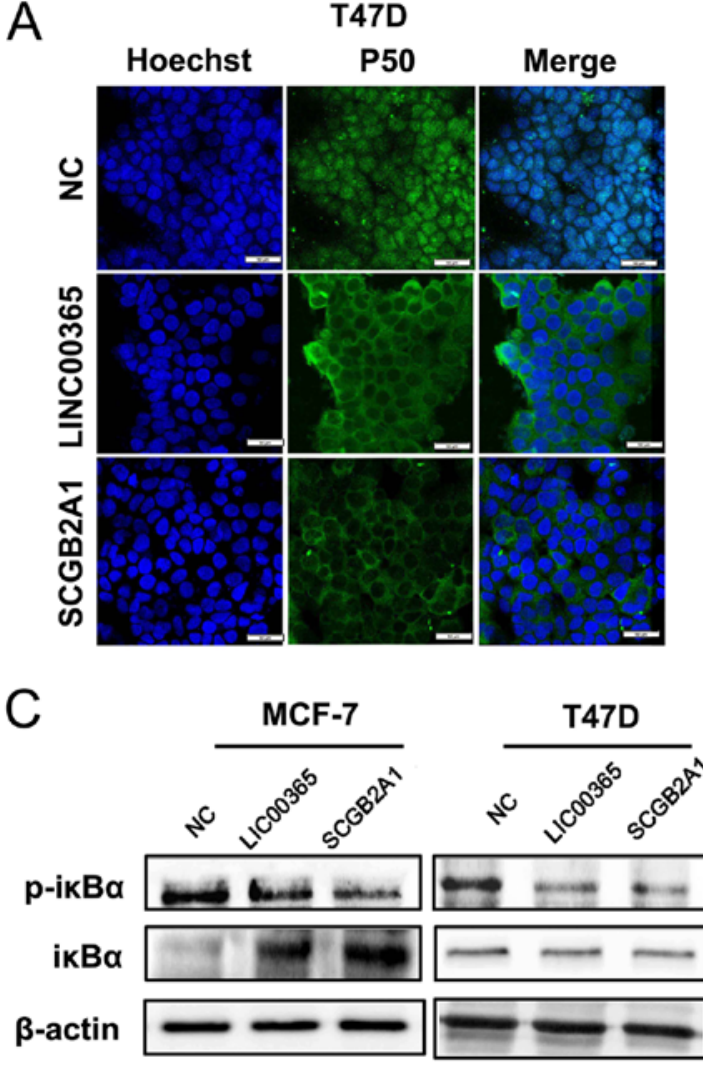

$\mathrm{E}$
B

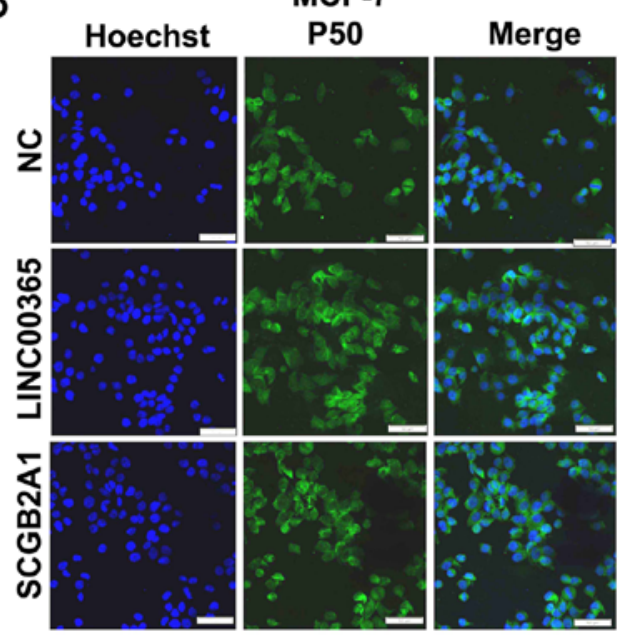

D

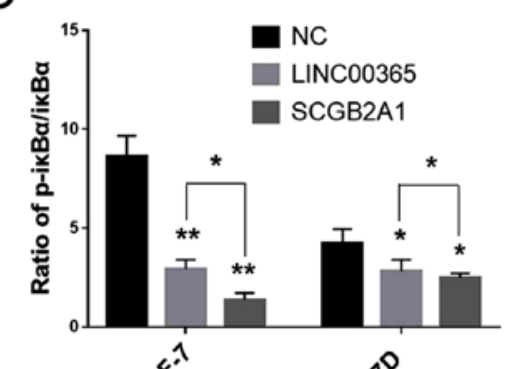

F

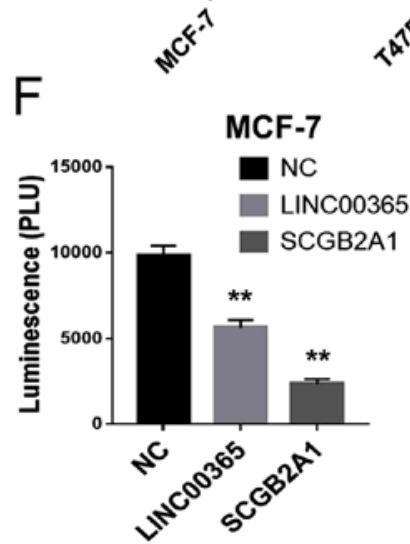

Figure 4. LINC00365 regulates SCGB2A1-mediated inhibition of the NF- $\mathrm{BB}$ signaling pathway. (A and B) Confocal images of changes in p50 expression in the nuclei of (A) T47D and (B) MCF-7 breast cancer cells following overexpression of LINC00365 and SCGB2A1. Scale bar, $50 \mu \mathrm{m}$. (C) Following overexpression of

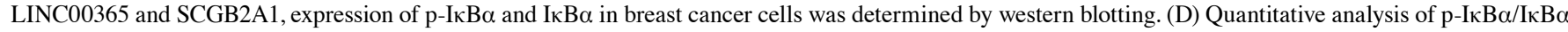
protein levels. (E and F) Detection of NF- $\kappa B$ signaling pathway activity in (E) T47D and (F) MCF-7 cells by dual luciferase reporter assay. ${ }^{*} \mathrm{P}<0.05$ and ${ }^{* *} \mathrm{P}<0.01$ vs. NC unless indicated otherwise. SCGB2A1, secretoglobin family 2A member 1; IкB $\alpha, \mathrm{NF}-\kappa \mathrm{B}$ inhibitor $\alpha$; p-, phosphorylated; NC, negative control.

and localization of p50 follwoing LINC00365 or SCGB2A1 overexpression; the results demonstrated that, compared with that in the $\mathrm{NC}$ group, the expression of p50 protein in the nucleus was decreased following transfection with LINC00365 or SCGB2A1 in MCF-7 and T47D cells compared with NC (Fig. 4A and B).

Dual luciferase reporter gene assay was performed to confirm the effects of LINC00365 or SCGB2A1 overexpression on the NF- $\mathrm{kB}$ signaling pathway. Consistent with results of the immunofluorescence assay, transcriptional activity of NF- $\kappa \mathrm{B}$ was decreased in the transfected groups (Fig. 4E and F). In addition, qPCR was used to detect apoptosis and viability-related genes regulated by NF- $\mathrm{BB}$. The results demonstrated that the levels of the anti-apoptotic gene cellular inhibitor of apoptosis 1 and the pro-survival genes
CXCL-8 and TGF- $\beta$ were decreased in the transfected groups in the two cell lines, which suggested that LINC00365 and SCGB2A1 may control cell viability through the regulation of NF- $\kappa B$ signaling (Fig. 5A-F).

\section{Discussion}

Increasing evidence has demonstrated that IncRNAs are involved in cell viability, apoptosis, migration and invasion in breast cancer (32). Certain lncRNAs have been identified as tumor-driving oncogenes, whereas others are tumor suppressors $(33,34)$. Therefore, IncRNAs can be used as biomarkers for diagnosis and prognosis of breast cancer and as potential therapeutic targets (35). The results of the present study 

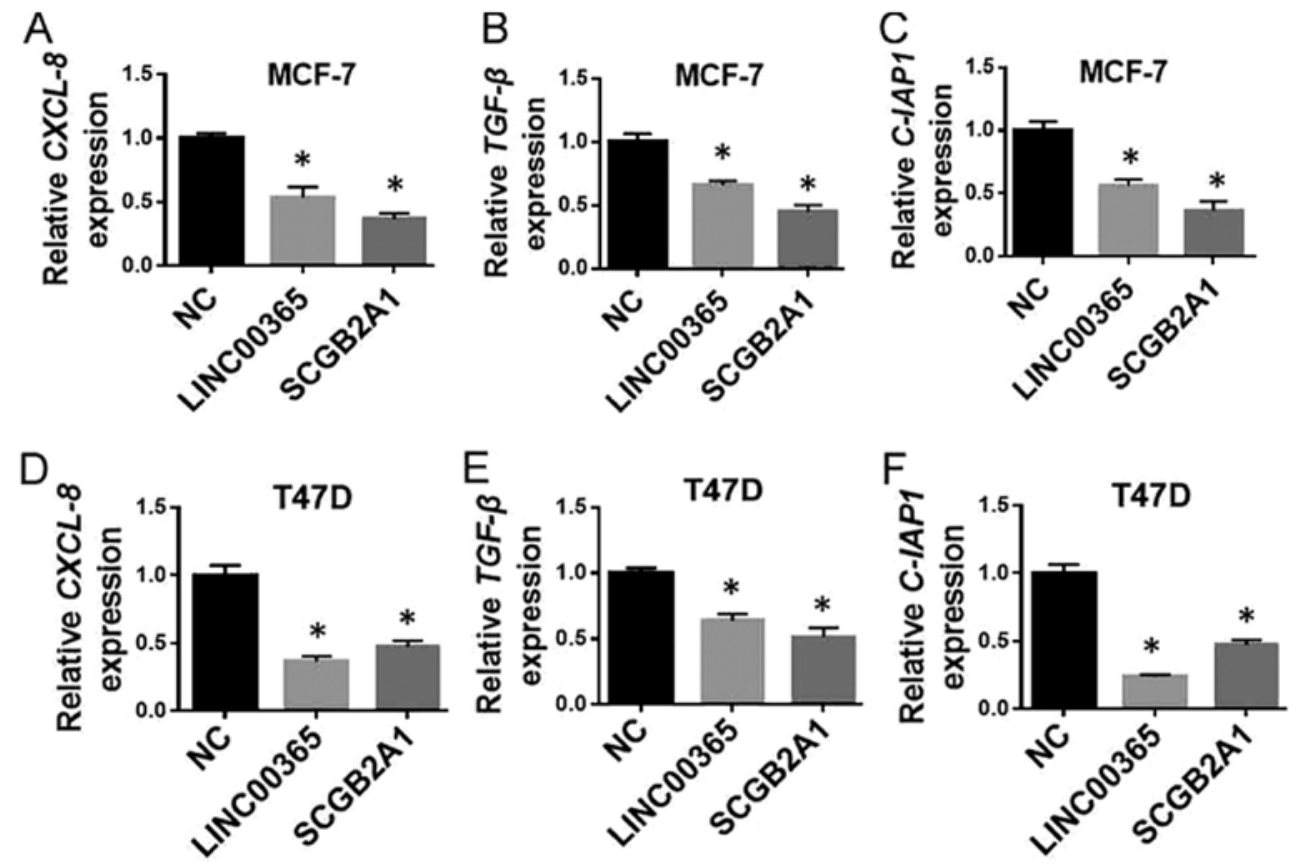

Figure 5. Regulation of downstream genes by decreased NF- $\mathrm{kB}$ activity. (A-F) Changes of (A and D) CXCL-8, (B and E) TGF- $\beta$ and (C and F) c-IAP1 in MCF-7 and T47D breast cancer cells following overexpression of LINC00365 and SCGB2A1, as determined by quantitative PCR. "P<0.05 vs. NC. CXCL-8, C-X-C motif chemokine ligand 8 ; TGF- $\beta$, transforming growth factor $\beta$; c-IAP1, cellular inhibitor of apoptosis 1 ; SCGB2A1, secretoglobin family $2 \mathrm{~A}$ member 1 ; NC, negative control.

demonstrated that overexpression of lncRNA LINC00365 resulted in the upregulation of SCGB2A1. In addition, the LINC00365-SCGB2A1 axis inhibited the activation of the $\mathrm{NF}-\mathrm{\kappa B}$ signaling pathway in breast cancer cells, subsequently inhibiting cell viability and inducing apoptosis. Therefore, the LINC00365-SCGB2A1 axis may be a potential therapeutic target in breast cancer (36).

Our previous study hypothesized that the level of LINC00365 may correlate with that of the protein encoded by its potential regulatory target gene SCGB2A1 in gastric cancer (3). Recently, SCGB2A1 was detected in the secretory mucosal epithelia of the breast, uterus and lacrimal glands, and it is known to be expressed in the prostate (10). Wong et al (5) also demonstrated that SCGB2A1 was upregulated upon bisphenol A treatment in the rat prostate, suggesting its involvement in prostate development. Other studies have reported that both LINC00365 and SCGB2A1 are expressed at a low level in primary breast cancer and occult breast metastatic tissues $(3,10)$. It may be speculated that LINC00365 and SCGB2A1 may be inhibited by cancer-related factors and pathways, which would result in a trend for low expression. Based on the analyses of clinical breast cancer specimens and breast cancer cell lines MCF-7 and T47D, the results of the present study demonstrated that SCGB2A1 may participate in tumor development by affecting the activation of NF- $\mathrm{KB}$ signaling.

$\mathrm{NF}-\kappa \mathrm{B}$ is constitutively activated in a number of tumors and is considered to be a key factor in cancer development $(37,38)$. $\mathrm{NF}-\kappa \mathrm{B}$ is involved in gene regulation, the activation of which promotes cell viability and inhibits cell death (39). Its normally localized in cytoplasm in an inactive form and binds

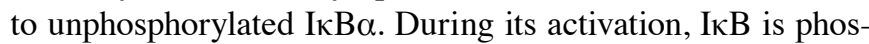
phorylated and thus dissociates from NF- $\mathrm{kB}$; subsequently, $\mathrm{NF}-\mathrm{kB}$ is transferred from the cytoplasm to the nucleus, where it regulates gene expression (40). The results of the present study indicated that overexpression of either LINC00365 or SCGB2A1 downregulated the activity of the NF- $\mathrm{KB}$ signaling pathway. Interestingly, compared with LINC00365 transfection, SCGB2A1 exhibited stronger effects on NF- $\kappa B$ signaling pathway inhibition. Similarly, pro-apoptotic effects induced by SCGB2A1 overexpression were stronger compared with those induced by LINC00365 in T47D and MCF-7 breast cancer cells. These results indicated that LINC00365 may have other target genes that impair the effects caused by SCGB2A1. In addition, following overexpression of LINC00365 or SCGB2A1, the apoptotic rate of MCF-7 cells was notably higher compared with that of T47D cells, which suggested that different breast cancer cell lines may have different sensitivity to LINC00365.

Apoptosis can be triggered in a cell through either the caspase-mediated intrinsic or extrinsic pathway (41). The extrinsic apoptotic pathway (death receptor-dependent) is initiated by the interaction of death receptors exposed on the cell surface; the more intensely characterized signaling systems of death receptor-ligands include recombinant Human TNF Receptor Type 1-TNF $\alpha$, factor associated suicide (FAS) (CD95, APO-1)-FasL, TNF related apoptosis inducing ligand related receptor 2 TRAILR2 (DR5)-TRAIL, and TRAILR1 (DR4)-TRAIL (41). By contrast, the intrinsic apoptotic pathway (mitochondrion-dependent) is mediated by intracellular signals that gather at the mitochondrial level in response to stress. The intrinsic pathway is frequently regulated by the Bcl-2 family of intracellular proteins (42). This protein family controls the alteration of mitochondrial outer membrane permeabilization by regulating the intrinsic pathways that promote and inhibit apoptosis (43). Bax serves a key role in the mitochondrial stress-induced apoptosis and can form a dimer with Bcl-2; this dimer enhances the permeability of 
the mitochondrial membrane and releases cytochrome $\mathrm{C}$ and $\mathrm{Ca}^{2+}$, leading to caspase activation (44). In MCF-7 cells, the Bcl-2/Bax dimer expression was decreased following overexpression of LINC00365 compared with that upon overexpression of SCGB2A1; however, the overall apoptotic rate was higher, which indicated that overexpression of LINC00365 may activate the extrinsic apoptotic pathway. In addition, following transfection with LINC00365 or SCGB2A1 overexpression vectors, the expression levels of $\mathrm{Bcl}-2$ family proteins changed significantly, but no notably change was observed in cleaved-caspase 3, which indicated that LINC00365 and SCGB2A1 may regulate additional pathways to counteract apoptosis in MCF-7 cells. In T47D cells, Bcl-2 family protein expression levels exhibited little change, but cleaved-caspase 3 expression was increased significantly, which indicated that LINC00365 and SCGB2A1 may regulate extrinsic apoptosis. Therefore, although the LINC00365-SCGB2A1 axis may be used as a tumor marker or a therapeutic target, it exhibits certain differences among breast cancer cell lines. However, the LINC00365-SCGB2A1 axis may affect biological characteristics of breast cancer by regulating the $\mathrm{NF}-\mathrm{\kappa B}$ signaling pathway.

The results of the present study suggested that LINC00365 may be a potential lncRNA biomarker for breast cancer treatment. Further in vivo experiments and clinical studies are needed to verify the activation of the LINC00365-SCGB2A1 axis. Based on existing reports, LINC00365 may further regulate SCGB2A1 by chromatin modifications, which will be verified in our future studies $(45,46)$. In addition, in further studies, high-throughput sequencing will be used to explore other regulatory mechanisms.

In conclusion, the results of the present study revealed the involvement of the LINC00365-SCGB2A1 axis in breast cancer. This axis may inhibit breast cancer cell viability and promote apoptosis through the suppression of the NF- $\kappa \mathrm{B}$ signaling pathway. The LINC00365-SCGB2A1 axis may become a novel target of breast cancer therapy.

\section{Acknowledgements}

Not applicable.

\section{Funding}

The present study was supported by the National Natural Science Foundation of China (grant nos. 81672948, 81772794 and 81501982), Jilin Provincial Health Special Project (grant no. 2018SCZ021), Jilin Provincial Industrial Innovation Project (grant no. 2018C052-7) and Jilin University Bethune Plan B Projects (grant no. 2015222).

\section{Availability of data and materials}

The datasets used and/or analyzed during the present study are available from the corresponding author on reasonable request.

\section{Authors' contributions}

LZ, XZ, and SY obtained the data and created the first draft of the article. JS and XB obtained the clinical tissue samples and designed the study. LX and RT analyzed the experimental data. LZ, XY, and JS participated in the project design and article revision. $\mathrm{XB}$ and $\mathrm{XY}$ revised the manuscript. All authors read and approved the final version of the manuscript.

\section{Ethics approval and consent to participate}

This study was approved by the Ethics Committee of the China-Japan Union Hospital of Jilin University. Complete clinical data were available for the patients participating in the study, and written informed consent was obtained.

\section{Patient consent for publication}

Not applicable.

\section{Competing interests}

The authors declare that they have no competing interests.

\section{References}

1. Harbeck N and Gnant M: Breast cancer. Lancet 389: 1134-1150, 2017.

2. Gu G, Dustin D and Fuqua SA: Targeted therapy for breast cancer and molecular mechanisms of resistance to treatment. Curr Opin Pharmacol 31: 97-103, 2016.

3. Li Y, He Y, Han S and Liang Y: Identification and functional inference for tumor-associated long non-coding RNA. IEEE/ACM Trans Comput Biol Bioinform 16: 1288-1301, 2019.

4. Munakata K, Uemura M, Takemasa I, Ozaki M, Konno M, Nishimura J, Hata T, Mizushima T, Haraguchi N, Noura S, et al: SCGB2A1 is a novel prognostic marker for colorectal cancer associated with chemoresistance and radioresistance. Int J Oncol 44: 1521-1528, 2014.

5. Wong RL, Wang Q, Trevino LS, Bosland MC, Chen J, Medvedovic M, Prins GS, Kannan K, Ho SM and Walker CL: Identification of secretaglobin Scgb2a1 as a target for developmental reprogramming by BPA in the rat prostate. Epigenetics 10 : 127-134, 2015.

6. Bellone S, Tassi R, Betti M, English D, Cocco E, Gasparrini S, Bortolomai I, Black JD, Todeschini P, Romani C, et al: Mammaglobin B (SCGB2A1) is a novel tumour antigen highly differentially expressed in all major histological types of ovarian cancer: Implications for ovarian cancer immunotherapy. $\mathrm{Br}$ J Cancer 109: 462-471, 2013.

7. Becker RM, Darrow C, Zimonjic DB, Popescu NC, Watson MA and Fleming TP: Identification of mammaglobin $\mathrm{B}$, a novel member of the uteroglobin gene family. Genomics 54: 70-78, 1998.

8. Lu X, Wang N, Long XB, You XJ, Cui YH and Liu Z: The cytokine-driven regulation of secretoglobins in normal human upper airway and their expression, particularly that of uteroglobin-related protein 1, in chronic rhinosinusitis. Respir Res 12: $28,2011$.

9. Lacroix M: Significance, detection and markers of disseminated breast cancer cells. Endocr Relat Cancer 13: 1033-1067, 2006.

10. Zubor P, Hatok J, Moricova P, Kajo K, Kapustova I, Mendelova A, Racay P and Danko J: Gene expression abnormalities in histologically normal breast epithelium from patients with luminal type of breast cancer. Mol Biol Rep 42: 977-988, 2015.

11. Claerhout S, Lim JY, Choi W, Park YY, Kim K, Kim SB, Lee JS, Mills GB and Cho JY: Gene expression signature analysis identifies vorinostat as a candidate therapy for gastric cancer. PLoS One 6: e24662, 2011.

12. Li J, Li Z, Zheng W, Li X, Wang Z, Cui Y and Jiang X: LncRNA-ATB: An indispensable cancer-related long noncoding RNA. Cell Prolif 50, 2017.

13. Yan B, Yao J, Liu JY, Li XM, Wang XQ, Li YJ, Tao ZF, Song YC, Chen Q and Jiang Q: IncRNA-MIAT regulates microvascular dysfunction by functioning as a competing endogenous RNA. Circ Res 116: 1143-1156, 2015. 
14. Bhan A, Soleimani M and Mandal SS: Long noncoding RNA and cancer: A new paradigm. Cancer Res 77: 3965-3981, 2017.

15. Huarte M: The emerging role of lncRNAs in cancer. Nat Med 21: $1253-1261,2015$

16. Mao Z, Li H, Du B, Cui K, Xing Y, Zhao X and Zai S: LncRNA DANCR promotes migration and invasion through suppression of lncRNA-LET in gastric cancer cells. Biosci Rep 37: BSR20171070, 2017.

17. Wang J, Geng Z, Weng J, Shen L, Li M, Cai X, Sun C and Chu M: Microarray analysis reveals a potential role of LncRNAs expression in cardiac cell proliferation. BMC Dey Biol 16: 41, 2016.

18. Fan Q and Liu B: Identification of a RNA-Seq based 8-long non-coding RNA signature predicting survival in esophageal cancer. Med Sci Monit 22: 5163-5172, 2016.

19. Zhang X, Zhang G, Zhang H, Karin M, Bai H and Cai D Hypothalamic IKKbeta/NF-kappaB and ER stress link overnutrition to energy imbalance and obesity. Cell 135: 61-73, 2008.

20. Turco MC, Romano MF, Petrella A, Bisogni R, Tassone P and Venuta S: NF-kappaB/Rel-mediated regulation of apoptosis in hematologic malignancies and normal hematopoietic progenitors. Leukemia 18: 11-17, 2004.

21. Rogers C, Fernandes-Alnemri T, Mayes L, Alnemri D, Cingolani $G$ and Alnemri ES: Cleavage of DFNA5 by caspase-3 during apoptosis mediates progression to secondary necrotic/pyroptotic cell death. Nat Commun 8: 14128, 2017.

22. Zhou A, Scoggin S, Gaynor RB and Williams NS: Identification of NF-kappa B-regulated genes induced by TNFalpha utilizing expression profiling and RNA interference. Oncogene 22: 2054-2064, 2003

23. Kato T, Khanh VC, Sato K, Takeuchi K, Carolina E, Yamashita T, Sugaya $\mathrm{H}$, Yoshioka T, Mishima $\mathrm{H}$ and Ohneda O: Corrigendum to 'SDF-1 improves wound healing ability of glucocorticoid-treated adipose tissue-derived mesenchymal stem cells' [Biochem. Biophys. Res. Commun. 493/2 (2017) 1010-1017]. Biochem Biophys Res Commun 497: 464-465, 2018

24. Park JH, Seo YH, Jang JH, Jeong CH, Lee S and Park B: Asiatic acid attenuates methamphetamine-induced neuroinflammation and neurotoxicity through blocking of NF-kB/STAT3/ERK and mitochondria-mediated apoptosis pathway. J Neuroinflammation 14: 240, 2017.

25. Yan J, Xiang J, Lin Y, Ma J, Zhang J, Zhang H, Sun J, Danial NN, Liu $J$ and Lin $A$ : Inactivation of BAD by IKK inhibits $\mathrm{TNF} \alpha$-induced apoptosis independently of NF- $\beta \mathrm{B}$ activation. Cell 152: 304-315, 2013.

26. Taniguchi F, Uegaki T, Nakamura K, Mon KY, Harada T, Ohbayashi T and Harada T: Inhibition of IAP (inhibitor of apoptosis) proteins represses inflammatory status via nuclear factor-kappa B pathway in murine endometriosis lesions. Am J Reprod Immunol 79: 2018.

27. Chen Y, Decker KF, Zheng D, Matkovich SJ, Jia L and Dorn GW 2nd: A nucleus-targeted alternately spliced Nix/Bnip3L protein isoform modifies nuclear factor KB (NFKB)-mediated cardiac transcription. J Biol Chem 288: 15455-15465, 2013

28. Oeckinghaus A and Ghosh S: The NF-kappaB family of transcription factors and its regulation. Cold Spring Harb Perspect Biol 1: a000034, 2009.
29. Pires BR, Mencalha AL, Ferreira GM, de Souza WF, Morgado-Díaz JA, Maia AM, Corrêa S and Abdelhay ES: NF-kappaB is involved in the regulation of EMT genes in breast cancer cells. PLoS One 12: e0169622, 2017.

30. Tang Y, Wang Y, Kiani MF and Wang B: Classification, treatment strategy, and associated drug resistance in breast cancer. Clin Breast Cancer 16: 335-343, 2016.

31. Livak KJ and Schmittgen TD: Analysis of relative gene expression data using real-time quantitative PCR and the 2(-Delta Delta C(T)) method. Methods 25: 402-408, 2001.

32. Soudyab M, Iranpour M and Ghafouri-Fard S: The role of long non-coding RNAs in breast cancer. Arch Iran Med 19: 508-517, 2016.

33. Xue X, Yang YA, Zhang A, Fong KW, Kim J, Song B, Li S, Zhao JC and Yu J: LncRNA HOTAIR enhances ER signaling and confers tamoxifen resistance in breast cancer. Oncogene 35: 2746-2755, 2016

34. Jiang Q, Wang J, Wu X, Ma R, Zhang T, Jin S, Han Z, Tan R, Peng J, Liu G, et al: LncRNA2 target: A database for differentially expressed genes after lncRNA knockdown or overexpression. Nucleic Acids Res 43: D193-D196, 2015.

35. Boon RA, Jae N, Holdt L and Dimmeler S: Long noncoding RNAs: From clinical genetics to therapeutic targets? J Am Coll Cardiol 67: 1214-1226, 2016.

36. Chen KS, Lim JWC, Richards LJ and Bunt J: The convergent roles of the nuclear factor I transcription factors in development and cancer. Cancer Lett 410: 124-138, 2017.

37. Karin M: Nuclear factor-kappaB in cancer development and progression. Nature 441: 431-436, 2006.

38. Sun SC: The non-canonical NF-kappaB pathway in immunity and inflammation. Nat Rev Immunol 17: 545-558, 2017

39. Wong RS: Apoptosis in cancer: From pathogenesis to treatment. J Exp Clin Cancer Res 30: 87, 2011.

40. Huxford T, Huang DB, Malek S and Ghosh G: The crystal structure of the IkappaBalpha/NF-kappaB complex reveals mechanisms of NF-kappaB inactivation. Cell 95: 759-770, 1998.

41. Youle RJ and Strasser A: The BCL-2 protein family: Opposing activities that mediate cell death. Nat Rev Mol Cell Biol 9: 47-59, 2008.

42. Pistritto G, Trisciuoglio D, Ceci C, Garufi A and D'Orazi G: Apoptosis as anticancer mechanism: Function and dysfunction of its modulators and targeted therapeutic strategies. Aging (Albany NY) 8: 603-619, 2016.

43. Pena-Blanco A and Garcia-Saez AJ: Bax, Bak and beyond - mitochondrial performance in apoptosis. FEBS J 285: 416-431, 2018.

44. Ashkenazi A, Fairbrother WJ, Leverson JD and Souers AJ: From basic apoptosis discoveries to advanced selective BCL-2 family inhibitors. Nat Rev Drug Discov 16: 273-284, 2017.

45. Schmitt AM and Chang HY: Long noncoding RNAs in cancer pathways. Cancer Cell 29: 452-463, 2016.

46. Bierhoff $\mathrm{H}$ : Analysis of lncRNA-protein interactions by RNA-protein pull-down assays and RNA immunoprecipitation (RIP). Methods Mol Biol 1686: 241-250, 2018.

This work is licensed under a Creative Commons Attribution-NonCommercial-NoDerivatives 4.0 International (CC BY-NC-ND 4.0) License. 